\title{
DE LA POSIBLE RENOVACIÓN DE LA TEORÍA CRÍTICA EN FRANCIA ENTRE DESVENTURAS ACADÉMICAS Y TENSIONES BOURDIEU/ RANCIÈRE
}

Philippe Corcuff

\section{Resumen}

El lazo histórico en los sectores significativos de las ciencias sociales entre la crítica social y la emancipación parece estar en riesgo, tanto en el campo académico como en el político, particularmente en Francia actual. Es a partir de estas apuestas que se dibuja la perspectiva de una reconfiguración comprensivo-pragmática de una teoría crítica con apoyos emancipadores en las ciencias sociales. Para ello, se abordan alternativamente ciertas desventuras académicas actuales de las relaciones entre la crítica sociológica y la emancipación, y luego las tensiones entre la sociología crítica de Pierre Bourdieu y la filosofía de la emancipación de Jacques Rancière. La sociología de inspiración libertaria de Alfredo Errandonea es utilizada como un recurso en este marco.

Palabras clave: Alfredo Errandonea / emancipación / Jacques Rancière / Pierre Bourdieu / teoría crítica.

\begin{abstract}
Of the possible revival of a critical theory in social sciences in France, between academic misadventures and tensions Bourdieu/Rancière

The historical link in significant sectors of social sciences between social criticism and emancipation seems at risk, in the academic and political fields, quite particularly in France today. It is from these stakes that takes shape the perspective of a comprehensive-pragmatic reconfiguration of a critical theory with emancipators supports in social sciences. For that purpose, are alternately envisaged certain current academic misadventures of relationships between sociological criticism and emancipation, and then the tensions between Pierre Bourdieu's critical sociology and Jacques Rancière's philosophy of emancipation. Alfredo Errandonea's sociology, drawing from an anarchistic inspiration, is used as a resource in this case.
\end{abstract}

Keywords: Alfredo Errandonea / Emancipation / Jacques Rancière / Pierre Bourdieu / Critical Theory.

Philippe Corcuff. Profesor de Ciencias Políticas en el Instituto de Estudios Políticos de Lyon, Francia. Miembro del laboratorio de sociología CERLIS (Centro de Investigación de Vínculos Sociales, Centro Nacional para la Investigación Científica-Universidad de París V René Descartes-Universidad Sorbona Nueva].

E-mail: philippe.corcuff@sciencespo-lyon.fr 


\section{Introducción ${ }^{1}$}

Este artículo es parte de unos ensayos en curso sobre la reconfiguración de una teoría crítica en ciencias sociales ajustada a ciertas apuestas intelectuales y políticas del siglo XXI (Boltanski, 2009; Corcuff, 2012; Boltanski y Fraser, 2016). Se inscribe en el campo de la teoría crítica desarrollada a partir de los años 1929-1930 por la Escuela de Frankfurt, que liga la crítica social y el horizonte de la emancipación (Horkheimer, 1996), pero con una reformulación a la vez comprensivista y pragmática. A partir de este marco general, voy a explorar dos series de problemas que se interrelacionan: primero, las desventuras o desencuentros académicos en Francia hoy en día en las relaciones entre crítica sociológica y emancipación, y, segundo, las tensiones entre la sociología crítica de Pierre Bourdieu y la filosofía de la emancipación de Jacques Rancière.

Este doble esclarecimiento nos permitirá trazar en puntos suspensivos posibilidades de reasociación entre la sociología crítica y la emancipación. Y encontrará el apoyo inesperado de la sociología crítica de inspiración libertaria del uruguayo Alfredo Errandonea. Entiendo reasociación en un sentido no hegeliano: no se trata para mí de superar una contradicción en una entidad englobante superior, sino de reasociar preservando la conciencia de la tensión. Me he inspirado aquí en las críticas poco conocidas hechas a la dialéctica hegeliana por un pionero del anarquismo, Pierre-Joseph Proudhon, quien hizo de la antinomia, más que de la superación de las contradicciones, la categoría central (Proudhon, 1988, pp. 35-36 y 57) y formuló una pista interesante epistemológicamente con la expresión "equilibración de los contrarios" (Proudhon, 1997, p. 20; Corcuff, 2016a).

Este procedimiento supone como consideración previa la necesidad de precisar algunas características sobresalientes del contexto de las relaciones entre crítica social, donde la sociología crítica constituye un componente científico autónomo, y emancipación, concentrándome en el caso francés.

1 Este artículo fue escrito originalmente en francés por Philippe Corcuff y traducido al español por Marcos Supervielle (Departamento de Sociología, Facultad de Ciencias Sociales, Universidad de la República, Uruguay), tanto en lo que refiere al texto central del artículo de Corcuff como a las citas que este hace de otros autores. 
Propongo la hipótesis de que, en el período actual, existen perturbaciones que atraviesan la mirada clásica de la crítica social con orientación emancipadora, es decir, de la crítica social que apunta los aspectos negativos de los órdenes sociales existentes (el núcleo del gesto crítico) en el horizonte de un positivo llamado, en este caso, emancipación.

Entiendo emancipación en el sentido que este término comenzó a tomar en el curso del llamado Siglo de las Luces (siglo XVIII) en Europa y en Estados Unidos: una suerte de "salida de los hombres por fuera del estado de tutela", según la expresión de Immanuel Kant (1991, p. 43).

Hoy, mucho después del nacimiento del movimiento socialista y con los aportes de las ciencias sociales modernas, hablaremos de una salida de las dominaciones en la construcción de una autonomía individual y colectiva bajo el supuesto de ciertas condiciones sociales. Históricamente no todas las sociologías se inscriben en este tipo de articulación entre crítica y emancipación. Sin embargo, importantes corrientes de la sociología se han alimentado de este apareamiento; Karl Marx y la tradición marxista (por lo tanto, la Escuela de Frankfurt), por supuesto, pero también, bajo modalidades diferentes del socialismo reformista y republicano, Emile Durkheim y Marcel Mauss (Chanial, 2001, pp. 203-221).

Si estos lazos se han distendido hoy en día en el universo académico francés es por una pluralidad de razones, como comenzaremos a ver en esta primera parte del artículo. Sin embargo, no es solamente en las ciencias sociales que crítica social y emancipación han tendido a disociarse, sino también en el campo político.

El compás de espera estalinista de lo que se ha llamado "comunismo", largamente marcado por la caída del Muro de Berlín en 1989, ha aportado sobre este plano un conjunto de desencantos y dudas. Por otro lado, la conversión, en los años ochenta, de numerosas organizaciones socialdemócratas al neoliberalismo económico, bajo la forma de un social-liberalismo, también ha aportado su grano de arena. Las desventuras de los dos polos políticos principales a escala mundial que reivindican conllevar el lazo crítica socialemancipación, los partidos comunistas y partidos socialdemócratas, han tenido también sus efectos sobre esta pareja cada vez más tambaleante. Hacemos notar que esta disociación tendencial entre crítica social y emancipación en el campo intelectual y en el campo político ha dado hoy lugar, tanto en Europa (con, por ejemplo, el Frente Nacional de Marine Le Pen en Francia, y más allá, con remiendos ideológicos ultraconservadores fuertemente vivaces) como en Estados Unidos (con Donald Trump), a tentativas de reasociaciones ultraconservadoras entre crítica social y discriminación (xenofobias, sexismos, homofobias, etcétera) en un marco nacionalista (Boltanski y Esquerre, 2014; Corcuff, 2014). 
Una apuesta a la vez sociológica y política importante en el período, por lo tanto, consistiría en intentar rearticular el plano negativo (la crítica social en el sentido restringido del término) y el plano positivo (la perspectiva de la emancipación), sin descuidar, sin embargo, las tensiones entre los dos polos, cuyas ubicaciones actuales nos han permitido ser más conscientes.

\section{Desventuras académicas de la sociología en Francia}

La tendencia actual de la ultraespecialización de las ciencias sociales, en la lógica de los recortes disciplinarios y subdisciplinarios, la rutinización de los objetos de estudio más valorizados y las diferentes modalidades de conformismo académico que le son asociadas participan en la disociación silenciosa, en Francia, entre la sociología y las referencias explícitas a la emancipación.

El uso corporativo relativamente extendido de la referencia a la neutralidad axiológica, poco argumentado epistemológicamente pero que funciona más bien como un "gri-gri" que supuestamente ha desechado la falta de cientificidad proveniente del exterior, refuerza esta disociación y contribuye a hacerla poco consciente.

\section{La neutralidad acrítica de Nathalie Heinich}

La socióloga del arte Nathalie Heinich es una de las representantes de la sociología que ha intentado argumentar sistemáticamente en Francia la cuestión de la neutralidad axiológica, inspirada en Max Weber (Weber, 1965b), a través de su libro Lo que el arte le aporta a la sociología, de 1998, donde defiende "una neutralidad comprometida" (Heinich, 1998, pp. 71-72) pretendiendo suspender "todo discurso sobre la naturaleza o el valor de las cosas" (Heinich, 1998, p. 77), "asociado a una postura crítica" (Heinich, 1998, pp. 23-29). Sin embargo, uno encuentra en ella numerosos distanciamientos con respecto a los matices y las tensiones que abordan los análisis de Weber (Corcuff, 2016b).

Weber combate inicialmente la tesis de una ciencia social "sin presupuestos", porque solo "una porción de la realidad singular tiene interés y significación a nuestros ojos, y esto porque solamente esta porción está en relación con las ideas de valores culturales con las que nosotros abordamos la realidad concreta" (Weber, 1965a, p. 163). De aquí proviene la idea de una "relación a los valores" en la "selección y la formación de los objetos de una investigación científica" (Weber, 1965b, p. 434), en tensión en él con la necesidad de poner distancia con respecto a los juicios de valor. Lo que Julien Freund ha traducido inicialmente en francés como neutralidad axiológica (Wertfreiheit, en alemán) se presenta como "la exigencia extremadamente trivial que se impone al sabio o al profesor de realizar la distinción, porque estas son dos series de problemas simple- 
mente heterogéneas, entre la constatación empírica [...] y su propia toma de posición evaluativa de sabio que conlleva un juicio sobre los hechos" (Weber, 1965b, pp. 416-417).

La nueva traducción francesa propuesta por Isabelle Kalinowski del término Wertfreiheit como "no imposición de valores" (Kalinowski, 2005, p. 199) concuerda mejor con las sutilezas weberianas y nos orienta sobre otra pista, diferente de la de Heinich. Tendremos ante todo que dar cuenta de una advertencia de Weber (inicialmente realizada de manera reflexiva en relación con él mismo) contra los efectos de una posición de autoridad pedagógica. Es por ello que él no prohíbe a los sabios "expresar bajo las formas de juicio de valor los ideales que los animan" (Weber, 1965a, p. 133), siempre bajo la condición de "explicitar escrupulosamente a cada instante, a su propia consciencia y a la de los lectores, cuáles son los niveles de los valores que sirven a medir la realidad y cuáles son los que hacen derivar los juicios de valor" (Weber, 1965a, p. 133).

Weber aparece más orientado por una exigencia de distinción reflexiva entre análisis de los hechos y tomas de posición directamente axiológicas, y no por la tesis de una separación neta entre las dos. Su defensa del reclutamiento universitario de un anarquista como jurista, no a pesar de que era anarquista sino porque era anarquista y, por lo tanto, porque estaría "situado por fuera de las convenciones y supuestos que parecen evidentes a los otros" (Weber, 1965b, p. 411) y sería entonces susceptible de "descubrir en las intuiciones fundamentales de la teoría corriente del derecho una problemática que se escapa a todos aquellos para los que son demasiado evidentes" (Weber, 1965b, p. 411), se desarrolla en este sentido.

Nos hemos alejado del vocabulario de Heinich, "suspendiendo todo discurso sobre la naturaleza o el valor de las cosas" (Heinich, 1998, p. 77), vocabulario que retoma $(2017$, p. 18) y hasta endurece en un reciente libro consagrado a los valores, sin estar, por otro lado, mejor informada sobre los análisis que el mismo Weber realizara, movilizando siempre la misma caución: "regla de neutralidad axiológica" (2017, p. 18), la obligación de "neutralidad axiológica enunciada [...] por Max Weber" (2017, p. 58), un imperativo de "neutralidad axiológica" (2017, p. 106), "distinción radical" (2017, p. 107) o "una inducción a la neutralidad comúnmente considerada como la norma académica" (2017, p. 113).

Sin embargo, las esferas de los juicios de hecho y los juicios de valor no son eliminadas en Heinich por ella misma. Por ejemplo, el "rol social" que ella atribuye a "la neutralidad comprometida" en Lo que el arte le aporta a la sociología - un rol de mediación, de construcción de compromisos entre los intereses y los valores en juego, léase: "una refundación de un consenso" (Heinich, 1998, p. 81) - tiene ciertas connotaciones cercanas a una concepción normativa de la política y de la democracia, más próxima a la filoso- 
fía política de "la acción comunicacional" de Jürgen Habermas, orientada al consenso, que a la de la "incomprensión" de Jacques Rancière, que valoriza la conflictividad.

\section{La sociología crítica en la niebla: tensión entre Bernard Lahire y Geoffroy de Lagasnerie}

La cuestión de la neutralidad axiológica revela también tensiones en el campo de la sociología crítica francesa, y ello particularmente entre Bernard Lahire y Geoffroy de Lagasnerie.

Bernard Lahire es hoy una de las figuras de la sociología crítica de calidad, en el cruce de caminos de ambiciones teóricas y de investigaciones empíricas situadas en el corazón de la institución universitaria. En un libro que defiende legítimamente la sociología contra ciertas estigmatizaciones públicas (Lahire, 2016), propone "dos planos distintos: el primero no normativo, que sería el propio del conocimiento científico, por un lado, y el segundo, normativo, que sería el propio de la justicia, la policía y la prisión, etcétera" (2016, p. 35).

Este autor también moviliza a Weber pero de forma menos terminante que Heinich: "lo que Max Weber se esfuerza a justo título es en distinguir, a saber, el juicio de valor y la relación a los valores, si el investigador manifiesta siempre su relación a los valores a través de los objetos de estudio y la manera en la que concibe su trabajo en tanto que tal, que no consiste en decir qué está 'bien' y qué está 'mal”' (Lahire, 2016, p. 39). Sin embargo, en las conclusiones del libro, hace hincapié en el lazo histórico entre las ciencias sociales y el Siglo de las Luces, así como en el lazo actual entre ciencias sociales y democracia (2016, pp. 117-128). Por lo tanto, a fin de cuentas, la problematización de las relaciones complejas entre la cientificidad y lo normativo aparece de forma rudimentaria y poco explícita en el surco de la lectura corporativa dominante de la neutralidad axiológica.

Geoffroy de Lagasnerie es un joven pensador crítico, a la vez sociólogo y filósofo, situado en la periferia de la institución universitaria pero beneficiado de cierto reconocimiento público. En un libro de 2007 explicita algunos de sus apoyos epistemológicos. Critica ciertos desvíos de la autonomización científica que frenan la creatividad intelectual: "distribución universitaria de los poderes en tanto que factor de "conservadurismo" (De Lagasnerie, 2007, p. 61), "efectos de censura" (2007, p. 64), "rutinas disciplinarias" (2007, p. 75), "práctica autárquica y rutinaria de una disciplina" (2007, p. 82). Más recientemente, ha extendido estas pistas epistemológicas "a un número de valores del conocimiento y de la ciencia" (De Lagasnerie, 2016, p. 274), criticando el "crecimiento de la normalización universitaria y 
las lógicas disciplinarias" (2016, p. 273) asociadas a la "reaparición de una ética de la neutralidad" (2016, p. 275).

De Lagasnerie nos ayuda a captar mejor que una apertura de los campos de las ciencias sociales nos lleva a interrogaciones exteriores, a compromisos militantes o artísticos, entre otros, que pueden contrabalancear las tendencias conformistas generadas por las instituciones universitarias. Se sitúa, en este plan, en una cierta continuidad de las reflexiones del sociólogo americano Charles Wright Mills, invitando a "estimular la imaginación sociológica" (Mills, 2006, p. 216) contra la "burocratización de la sociología" (2006, p. 121), "las especializaciones arbitrarias de los departamentos universitarios" (2006, p. 137) y "los encerramientos disciplinarios" (2006, p. 143).

Sin embargo, De Lagasnerie baja la guardia en otro sentido, y debilita de esta forma su crítica a los puntos ciegos de la autonomización universitaria. Puede incluso flirtear con el relativismo epistemológico cuando escribe que es necesario "romper con la idea de que existirían diferencias de naturaleza y por lo tanto de valor entre un libro de investigación, un tratado filosófico, un ensayo, una intervención en la prensa, una manifestación o un volante político" (De Lagasnerie, 2007, p. 102) o producir una crítica demasiado unilateral del lugar de la investigación empírica en ciencias sociales (De Lagasnerie, 2016, pp. 267-296), como si no fuera uno de los dos pulmones del trabajo científico, ¡ciertamente contenido por las especializaciones disciplinarias en el "va y viene" con la elaboración teórica!

Sería más sutil y pragmático reconocer que, a la vez, las instituciones universitarias y las reglas disciplinarias protegen una cierta autonomía y un cierto rigor intelectual, frenando al mismo tiempo la imaginación sociológica a través de tendencias conformistas. Desde este punto de vista, sigo a Norbert Elias, para quien la distinción científica tiene por vocación transformarse en el polo dominante de la sociología, al mismo tiempo que se nutre de la variedad de las implicaciones de los investigadores de la ciudad, porque "su propia participación y su compromiso condicionan su inteligencia ante los problemas que deben resolver en su calidad de científicos" (Elias, 1993, p. 29).

\section{Del antinormativismo a la ruptura con la emancipación: Elsa Rambaud}

Un reciente artículo que apareció en la Revista francesa de ciencia politica (Rambaud, 2017) constituye un índice de estas derivas producidas en algunos sectores de las nuevas generaciones de los científicos sociales por una cierta radicalización del lugar común de la "neutralidad axiológica", en una lógica extrema de purificación sin cese, de un relanzamiento de lo "normativo" aprehendido como una contaminación eliminable, más que, como lo sugiere Weber, de una reflexividad sobre los componentes axioló- 
gicos y políticos difícilmente eliminables con el fin de mejor delimitar el dominio de la validez de los análisis producidos y, por lo tanto, de mejorar el rigor científico.

Elsa Rambaud es una joven doctora en ciencias políticas que ha efectuado una tesis sobre la organización no gubernamental humanitaria Médicos sin Fronteras (Rambaud, 2009). Pretende, en su texto de junio de 2017, proponer una superación de los supuestos límites compartidos entre la sociología crítica de Pierre Bourdieu y la sociología pragmática de Luc Boltanski, a pesar de sus diferencias y sus oposiciones. ¿Cuáles serían estos límites compartidos? Según la autora, guardarían lazos con las "Luces" (Siglo de las Luces), la emancipación, la revolución, la izquierda y la lucha de clases. Esto les impediría tomar por objeto una variedad de críticas de la sociedad que no corresponden a las "Luces", a la emancipación, a la revolución, a la izquierda y a la lucha de clases. Es por ello que sería necesario que las ciencias sociales terminasen definitivamente con las "Luces", la emancipación, la revolución, la izquierda y la lucha de clases, mientras que los sociólogos Bourdieu y Boltanski estarían todavía contaminados por un tal "normativo" que se debe purificar.

Sobre el plano teórico, la argumentación aparece marcada por errores de amplitud. Para comenzar, el artículo tiende a confundir en Bourdieu y Boltanski el nivel epistemológico de las propiedades de una teoría de las ciencias sociales, discutible en un espacio de debate científico, y el nivel del objeto analizado utilizando esa teoría. Sin embargo, una teoría que se nutre axiológicamente de una referencia a las "Luces", a la emancipación, a la revolución, a la izquierda y a la lucha de clases puede perfectamente tomar en serio, en el mundo social, críticas conservadoras e incluso racistas muy alejadas de sus propios valores (ver, por ejemplo, Boltanski y Esquerre, 2014). Por otro lado, es falso considerar que las obras de Bourdieu y Boltanski están siempre y uniformemente adosadas a las "Luces", a la emancipación, a la revolución, a la izquierda y a la lucha de clases. Textos muy diferentes entre sí son amalgamados de manera insuficientemente documentada.

Al mismo tiempo que muestra estas debilidades teóricas, el artículo de Rambaud conlleva riesgos políticos particulares en un momento en el que los esquemas ultraconservadores buscan dinamizar la crítica social sobre una base discriminatoria y nacionalista en los espacios públicos, aprovechando la fragilización de los lazos históricos entre la crítica social y la emancipación. Tenemos aquí una forma de ética irresponsable, la de forjar una noción inversa a la de una "ética de la responsabilidad", preocupada por las consecuencias de su acción sobre la realidad, postura avanzada por Weber (2003, pp. 192-193). Esta ética de la irresponsabilidad parece ser producida por la generalización abusiva y mal construida de una exigencia, localizada 
y legítima, de extensión del espacio de las críticas en las ciencias sociales. Esto podría contribuir a distender un poco más los lazos entre crítica social y emancipación, facilitando involuntariamente el proceso ultraconservador a través de una garantía intelectual suplementaria.

El muy estimulante trabajo científico de Rambaud, desarrollado sobre Médicos sin Fronteras, no necesitaba, sin embargo, todo este andamiaje, débil desde el punto de vista de la argumentación y peligroso políticamente, para consolidar la ampliación deseable del espacio de críticas analizadas por las ciencias sociales.

\section{Las tensiones entre la sociología de Bourdieu y la filosofía de Rancière}

La tensión entre la sociología crítica de Pierre Bourdieu y la filosofía de la emancipación de Jacques Rancière constituye una expresión singular de los problemas encontrados en la primera parte de este artículo. Pero una expresión desplazada, porque al integrar el punto de vista de la filosofía de la emancipación, este nos permite ampliar la mirada en relación con un punto de vista únicamente interno a la sociología. Al hacerlo, nos ayuda a encontrar pistas en cuanto a la reasociación crítica sociológica-emancipación, con la ayuda de Alfredo Errandonea, así como con la de Claude Grignon y Jean-Claude Passeron.

\section{La tensión Bourdieu/Rancière a nivel global}

Pierre Bourdieu ha renovado profundamente las teorías críticas de la dominación en un sentido que podríamos calificar de "posmarxista", en la medida en que descodifica una pluralidad de formas de dominación (Corcuff, 2009). Dos conceptos constituyen los ejes de su aproximación sociológica a la dominación: habitus (las relaciones sociales en el cuerpo y en las cabezas) y campos (las relaciones sociales en las instituciones concebidas a través de una visión espacial). Con el habitus, Bourdieu sigue las trazas no conscientes de las dominaciones sociales de los cuerpos y las cabezas de los individuos.

Citaré un pasaje significativo sobre el plan de su libro:

"El reconocimiento práctico por el cual los dominados contribuyen muchas veces sin quererlo, a veces contra su voluntad, a su propia dominación, aceptando tácitamente por anticipado los límites impuestos, toma muchas veces la forma de la emoción corporal (vergüenza, timidez, ansiedad, culpabilidad) [...] Ella se traiciona en las manifestaciones visibles como ruborizarse, tener bloqueos verbales, brusquedades, temblores, tantas maneras y tantas otras maneras de someterse, a pesar de sí mismo y de su cuerpo, defendiéndose del juicio dominante". (Bourdieu, 1997, p. 203) 
Bourdieu pone aquí en evidencia cómo las experiencias sociales de dominación se imprimen inconscientemente en los cuerpos, escapando a la voluntad de los individuos al límite de bloquear sus capacidades. Las constricciones exteriores de los campos sociales organizados según los modos de dominación específica (en los campos económicos, políticos, culturales, etcétera) vienen a redoblar los pesos interiorizados de las opresiones. Sin embargo, algunos impensados desde el ángulo de la dominación sobre las relaciones sociales son puestos en relieve por la filosofía de Jacques Rancière a partir de una perspectiva radicalmente democrática de emancipación. En una entrevista publicada en 2009, Rancière nos ayuda a clarificar el problema de las relaciones entre teorías críticas de la dominación y filosofías de la emancipación, en un cuestionamiento de la crítica situacionista de "la sociedad del espectáculo" de Guy Debord y de la sociología crítica de Pierre Bourdieu.

Sostiene lo siguiente:

“[...] esta forma de toda la crítica marxista revolucionaria ha absorbido un cierto número de presupuestos imaginarios; hay los activos y los pasivos; hay aquellos que miran y aquellos que saben. Lo que en términos generales quiere decir: hay aquellos que son capaces, y aquellos que no lo son: A partir de allí, hay múltiples estrategias posibles [...] se piensa que se hace necesaria una vanguardia que reúna a la gente capaz para poder poner en la cabeza de los incapaces los medios para zafarse". (Rancière, 2009, p. 622)

Para Rancière, los filósofos de la emancipación partirían de la posibilidad de la igualdad, apoyándose sobre las capacidades de los oprimidos, mientras que varias teorías críticas partirían de la desigualdad y, por lo tanto, supondrían que los oprimidos son completamente "incapaces".

Por lo tanto, tendríamos el riesgo de que la dominación tome todo el espacio en las teorías críticas, al ver a los dominados como totalmente sometidos a la dominación y como "alienados". En esta invasión desigualitaria de los pensadores críticos, incluso los esfuerzos de emancipación de los dominados tenderían a ser descriptos como efectos de "manipulación" o de una "recuperación" por "el sistema" o, incluso, como una reproducción poco hábil de los estereotipos dominantes. Los oprimidos serían así definitivamente encerrados en las jaulas de hierro de la dominación, y su emancipación, a pesar de ser señalada (tanto por Debord como por Bourdieu), sería políticamente imposible, porque sería continuamente postergada bajo el pretexto de la presión de la astucia de la dominación. Como Rancière, indica: "Siempre el amo guarda bajo el codo un saber, es decir una ignorancia del alumno" (Rancière, 2004, p. 38), haciendo recular al infinito el momento de igualdad y emancipación. 
Es aquí que aparece el deslizamiento subrepticio y frecuente del verbo pronominal emanciparse (del tipo "la emancipación de los trabajadores será obra de los propios trabajadores" en los estatutos de 1864 de la Asociación Internacional de los Trabajadores, escritos por Marx) al verbo transitivo emancipar (como amos que pueden emancipar esclavos en la América esclavista). Este desplazamiento, muchas veces de forma no consciente, hacia una aproximación tutelar de la emancipación pudo tomar diferentes figuras históricas en el campo político: ayer, el instructor republicano que emancipaba los prejuicios de la "tradición" o la vanguardia revolucionaria leninista, hoy, el denunciador altermundista de la "propaganda mediática" a fin de guiar al ciudadano fuera de la "caverna", la feminista que pretende liberar a las mujeres musulmanas veladas o las prostitutas contra ellas mismas o el profeta ecologista en los primeros puestos de la "desalienación" en relación con la "sociedad de consumo".

Estos análisis llevan a Rancière a abandonar el terreno teórico-crítico de la dominación. Escribe en este sentido: "La inteligencia colectiva de la emancipación no es la comprensión de un proceso global de contención. Ella es la colectivación de las capacidades invertidas en esos escenarios de disenso" (Rancière, 2008, p. 55).

De alguna manera, elige la filosofía de la emancipación contra la crítica de la dominación. Sin embargo, ¿la emancipación no supone en sí misma una referencia al menos implícita a la dominación, porque se trata de una emancipación de la dominación? ¿Pero por qué negarse a sostener algo sobre uno de los dos polos de esta cupla? ¿Podemos verdaderamente pensar la emancipación sin ninguna referencia a la dominación? Parece difícil.

Si Rancière esclarece los puntos ciegos de Bourdieu, recíprocamente, Bourdieu esclarece los puntos ciegos de Rancière, sobre todo cuando el sociólogo señala la manera en la que las dominaciones se insinúan inconscientemente en los cuerpos paralizando las capacidades de los individuos bajo formas no controladas, como la vergüenza o el sonrojarse.

Después de la descodificación global de la tensión entre la sociología de Bourdieu y la filosofía de Rancière, voy a proponer una modalidad particular, entre otras, para afinar el análisis.

\section{Una alodoxia ambivalente entre Bourdieu y Rancière}

En La distinción, Bourdieu adelanta la noción de alodoxia tomándola del griego antiguo: allo, del griego allos, es decir, "otro", y doxia, del griego doxa, "opinión" (Bourdieu, 1979, pp. 370-371, 377 y 538). Para Bourdieu se trata de un error de identificación, instrumentado por criterios sociales inadaptados en relación con la jerarquía cultural instituida. Es, por lo tanto, una noción marcada por la sombra del desconocimiento, que señala el 
carácter inhábil culturalmente de aquellos que son exteriores al círculo de legitimidad cultural dominante aun manifestando una "buena voluntad cultural". Esto apunta a la pequeña burguesía, pero puede también concernir a ciertos sectores de las clases populares (en particular, las autodidactas y las militantes). La alodoxia se presenta entonces como "desprecio y reino de las imitaciones". Es, por ejemplo, confundir la imitación del cuero con el cuero.

A la inversa, para Rancière, los itinerarios de los primeros enunciadores de la palabra de los obreros, en torno a 1830, ponen en evidencia que esta alodoxia no es lo que condena al encierro en la jaula de hierro de la dominación, sino lo que permite abrir un proceso de emancipación. Escribe lo siguiente: "los primeros militantes obreros han comenzado por tomarse por poetas o caballeros, curas, o dandys. La alodoxia es la única vía de la heterodoxia. Pasiones prestadas usando las únicas palabras que hacen posible la reapropiación: palabras prestadas" (Rancière, 1983, p. 286).

Es por ello que el procedimiento del sociólogo crítico es puesto en duda como forma de desvío eternizante en relación con lo que estigmatiza, como una suerte de imitación de la emancipación.

La postura sociológica instauraría una sospecha, "dondequiera que se encontrara se tendría una imagen de compartir y de equívoco" (Rancière, 1983, p. 288). Para Rancière, "la crítica sociológica dice del reino eterno de la pequeña diferencia [...] para negar que el sujeto de la democracia no puede alcanzarla nunca" (Rancière, 1983, p. 305). Me apoyaré sobre un ejemplo tomado de una investigación de campo para aclarar este punto al que llamo "Pierre Dubois, el infinito y el mal de estómago".

Este caso empírico es tomado de la investigación de campo realizada para mi tesis Una etnografia local del sindicalismo ferroviario (Corcuff, 1991). Se trata de un extracto de una entrevista semiestructurada con Pierre Dubois (realizada en abril de 1986), entonces de 58 años, militante sindical jubilado. Su padre había comenzado como operario y terminado como agente de conducción en el ferrocarril. Pierre tenía un certificado de aptitudes profesionales de ajustador, luego había recibido una formación de la empresa de ferrocarriles y había debutado como obrero profesional. Después se volvió agente de conducción, para culminar como cuadro profesional, jubilándose a los 52 años. Jubilado retomó sus estudios, al inicio realizando cursos nocturnos en la Bolsa de Trabajo y luego inscribiéndose en la universidad, donde obtuvo un diploma de comunicaciones (periodista). Léase de manera crítica "el intelectual" en relación con "el espíritu práctico", en referencia a su experiencia universitaria tardía:

"El intelectual puro, digamos, y hay mucho vapor en esto, tú ves, es una nebulosa para encontrar la idea dominante, la idea que se va a concretizar y que va 
a dar algo [... Dicho de otra manera, no es suficientemente, este..., no tiene un espíritu suficientemente pragmático. [...] Un espíritu que se pierde en teorías, y posibilidades y combinaciones. Entonces digo 'no'. Entonces, aquel que tiene un espíritu práctico se dice 'bueno, no vale la pena, ¿eh?' y entonces tienes el campo de reflexión operacional, que queda canalizado, entonces sí, en ese momento, se va rápido”. (Entrevista a Pierre Dubois, 1986)

Sin embargo, en otro momento reconoce la importancia del "trabajo de reflexión" y califica de "benéficos en el plano personal" sus dos años de vida universitaria. Para él, por otro lado, esta dimensión tiene tendencia "a faltar en el mundo obrero". Pero a las limitaciones obreras responden las limitaciones de los intelectuales, de alguna manera simétricas.

El peligro para el intelectual sería entonces "perderse" en una multiplicidad de "posibilidades" y de "combinaciones", en donde hay la necesidad de "canalizar". Para Pierre Dubois, "el espíritu práctico" surge de su experiencia familiar, profesional y militante, y constituye, entonces, la barrera frente a los vértigos de la reflexión intelectual, que pueden llevarlo hasta la angustia:

"Me gustaría mirar la astronomía, pero eso desemboca en una reflexión sobre el infinito, pero sobre eso no puedo, pierdo mi equilibro, ¿eh?, me da vuelta la barriga, no puedo. El infinito, veamos, después de la tapa de una caja hay eso [me muestra una caja], y después está el vacío, y después... Entonces imagine el infinito, lo que no termina nunca, lo que para mí es inaccesible al ser humano, yo no puedo [...] Entonces ahí no, ¡no! Ahí me enfermo, enfermo, me volvería loco, ¿eh? Es verdaderamente un punto, digo, ¡que me molesta mucho!”. (Entrevista a Pierre Dubois, 1986)

Sería fácil interpretar el discurso de Pierre Dubois, a la manera de Bourdieu, como alodoxia, en el sentido de ser un desprecio, ligado a una relación autodidacta con el trabajo intelectual, por ejemplo, en la conexión hecha entre la "reflexión filosófica" y el "mal de estómago". Pero esto sería un allanamiento miserabilista (en el sentido que le dan Claude Grignon y Jean Claude Passeron, sobre el que volveré) por las palabras de las experiencias y de las relaciones con el mundo manifiesto. ¿Y si retomamos en serio la manera en que Pierre Dubois construye una relación singular con el mundo intelectual? No se juega con las tensiones existentes entre el polo "práctico" y el polo "intelectual”, ¿un desplazamiento de la jerarquía cultural dominante tradicional?

Podemos observar entonces, a través de tanteos, una lógica emancipatoria, pasando por "préstamos", como dice Rancière, e hibridaciones. Nos alejamos de la tendencia legitimista de la noción de alodoxia en Bourdieu, siempre lista para desconfiar de la autenticidad intelectual de los discursos populares, autodidácticos y pequeñoburgueses. 
No estamos, sin embargo, situados en mundos irónicos, con el riesgo de olvidar el peso de las constricciones que genera la dominación. Parece, por lo tanto, oportuno el integrar una parte de la verdad de la crítica sociológica, so pena de mal rendir cuenta de otras características de la experiencia de Pierre Dubois, que se filtran a través de su discurso. Este integra, por otro lado, de una manera cierta las constricciones de la dominación: hay allí a la vez distancia y reverencia en su relación con el mundo intelectual. Y Pierre Dubois se reconoce sinceramente poco hábil en relación con los recursos y procedimientos de un mundo que no es el suyo de origen. El corte inicial de los mundos sociales es claramente sentido. Y, además, la angustia física frente al infinito en la reflexión intelectual constituye una marca corporal de la brecha con este universo intelectual. Con precisión, Bourdieu ha insistido frecuentemente en la importancia del cuerpo en las relaciones con las normas dominantes y los universos sociales más alejados. He citado, precisamente, un pasaje significativo de las Meditaciones pascalianas sobre el carácter involuntario de "la emoción corporal (vergüenza, timidez, ansiedad y culpabilidad)".

Préstamos y mestizajes emancipadores, por una parte, restricciones impuestas por la dominación, por otra: no deberíamos tener que doblar uno sobre otro para entender la ambivalencia de la experiencia, asumiendo, así, la tensión.

\section{Rancière/Bourdieu: dos aproximaciones a la emancipación}

En este punto del análisis, parece necesario detenerse un momento con el fin de comparar las figuras de la emancipación privilegiadas por Rancière y por Bourdieu. Para Rancière, la figura de la emancipación consiste en un sinceramiento de la división social, sobre todo por los préstamos tomados de los medios dominantes, estableciendo la posibilidad de una igualdad entre universos sociales marcados por la desigualdad. De esta forma, "cuando una lógica supuestamente natural de la dominación es traspasada por el efecto de esa igualdad", habría emancipación, adelanta (Rancière, 1995, p. 37). Y agrega que la emancipación, entonces, tendría que ver con "las brumas de la frontera" (Rancière, 2008, p. 26).

Hay también pistas sobre la emancipación en Bourdieu, aunque más marginales que en Rancière (de quien es uno de sus temas principales) y menos visibles en la economía de su sociología. Bourdieu se inspira en el esquema de la libertad relativa al conocimiento de las determinaciones en Baruch Spinoza (1965, parte III, proposición II, escolia, pp. 139-140 y parte V, proposición X, escolia, pp. 315-316). La emancipación, entonces, tiene que ver principalmente con el conocimiento de los propios determinismos sociales, y ello tanto a nivel individual como colectivo. 
Estas dos figuras de la emancipación no son necesariamente excluyentes la una con respecto a la otra. Podemos pensar, en el caso de Pierre Dubois, que las marcas emancipadoras pueden enriquecerse a través de un conocimiento desculpabilizante de las limitaciones contra las que choca. Bourdieu nos habla en este sentido, en el post-scriptum de la obra colectiva La miseria del mundo, de la posibilidad de imputar el sufrimiento a causas sociales y de ser así "disculpados" (Bourdieu, 1993, p. 944). Pero no debería ocurrir que la descodificación crítica de los determinismos sociales se trague el conjunto de la experiencia en la dinámica de una sospecha generalizada. ¡Nuevamente una tensión!

\section{Una iluminación desconocida en Europa: la sociología crítica de Alfredo Errandonea}

Figura desgraciadamente desconocida en Europa, el sociólogo uruguayo Alfredo Errandonea ha abierto senderos teóricos al asociar firmemente la crítica teórica y la emancipación como compromisos político-éticos y la autonomía del conocimiento científico. Tomó distancia del marxismo en una referencia original al anarquismo (Errandonea, 2003; Wschebor, 2003; Prieto, 2003). Errandonea nos permite abrir un paréntesis sugestivo en relación con el debate franco-francés.

Ha contribuido a afinar herramientas de la sociología crítica que se desprenden de la frecuente hegemonía teórica marxista en el siglo XX, tanto en la crítica social universitaria como en la crítica militante. En su libro Explotación y dominación (Errandonea, 1972), el autor evalúa los aportes y los límites de la noción marxista de explotación para reinscribirla en el seno de un concepto más amplio de dominación, a la que entiende "como configuración estructural de relaciones asimétricas" (1972, p. 51). Al hacerlo se separa de la pendiente economicista, y pone al poder en el centro del análisis, en tanto que "unos deciden lo que implica a los otros y/o a todos" (1972, p. 9). Para realizar este análisis, se apoya explícitamente en la ética anarquista, hablándonos en la introducción de la obra de "una perspectiva inspirada en valores libertarios" (1972, p. 9).

El desplazamiento teórico realizado por Errandonea se funda también directamente en las críticas anarquistas clásicas del poder y el Estado, en particular en Mijaíl Bakunin. Esto tiene que ver con el descentramiento de la sola explotación a fin de aprehender las características del Estado en el seno del capitalismo del siglo XX, como las de la Unión Soviética vista como "una organización despótica moderna" (1972, p. 41).

La crítica sociológica ampliada de Errandonea parece así arrimarse al horizonte de la emancipación. Este se presenta como el carburante éticopolítico del conocimiento científico, bajo la modalidad de la apertura de 
la "imaginación sociológica", cara a Charles Wright Mills (2006), a cuya sociología Errandonea hace referencia importante (Prieto, 2003, pp. 1213). Los recursos anarquistas ayudan a desplazar la mirada de las formas rutinarias de su época: en este caso, de la importancia de los esquemas marxistas en los años 1960-1970 en el seno de las ciencias sociales. Converge en la práctica con el argumento de Weber favorable a la contratación de un jurista anarquista como profesor de la universidad a causa, justamente, del descentramiento en relación con las problemáticas más admitidas en materia de derecho.

Este apoyo ético-político no desconoce los obstáculos políticos que también puede encontrar la sociología en la ruta científica. En Explotación y dominación Errandonea apunta la tendencia "ideológica" de un cierto dogmatismo marxista en su lógica de justificación de una "estrategia política" con efectos "oscurantistas", que se opone a todo "esfuerzo científico creativo" (Errandonea, 1972, pp. 70-71). Aquí Errandonea reconoce plenamente la autonomía del conocimiento científico. En este marco, la ética y la política pueden ser estimulantes para la imaginación científica y para los obstáculos del saber, la vigilancia epistemológica necesaria a esta "equilibración de los contrarios", donde el estilo de Proudhon hace su miel, según Rubén Prieto (2003, p. 13), de una "epistemología libertaria" que desconfía de construcciones teóricas cerradas y fijas, no abiertas a preguntas nacidas en la propia investigación. Está de acuerdo con el punto de vista de Weber cuando enfatiza la importancia de "reconocer hechos desagradables, hechos, quiero decir, que son desagradables para la propia opinión partidista" (Weber, 2003, p. 96). Se esboza así una configuración original de distanciamiento científico que se nutre de compromisos éticos y políticos.

\section{Una puesta en perspectiva epistemológica: Grignon y Passeron}

Después de este paréntesis heurístico uruguayo, volvamos a la tensión Bourdieu/Rancière. Para ponerlo en perspectiva en el marco de una epistemología sociológica, nos parece útil pasar por los análisis de Jean Claude Passeron y Claude Grignon en su libro de diálogo Le savant et le populaire (Grignon y Passeron, 1989). Passeron es el coautor de los primeros libros de Bourdieu y un gran epistemólogo contemporáneo de las ciencias sociales y Grignon también ha sido colaborador de Bourdieu.

Grignon y Passeron han puesto en evidencia que las aproximaciones sociológicas y literarias a las culturas populares (y a las culturas dominadas en general) tienden a oscilar entre dos derivas:

1. el legitimismo (o dominocentrismo, incluso miserabilismo), que solamente mira las prácticas populares jerarquizando las relaciones con las normas dominantes, socialmente las más legítimas, como si las activi- 
dades de sentido de los dominados pudiesen ser aprehendidas en su relación de dependencia con los dominantes.

2. el populismo, que sacraliza las culturas populares y dominadas en general como entidades dotadas de una total libertad, olvidando las características que estas deben a las relaciones de dominación (relaciones de clase, de género, poscoloniales, etcétera) en las cuales están insertas: es de notar que el término "populismo" tiene un sentido distinto a las acepciones más corrientes en América Latina.

Si seguimos las categorías de Rancière, el dominocentrismo partiría de la desigualdad y de las incapacidades de los oprimidos y el populismo partiría de la igualdad de las capacidades de los oprimidos.

Grignon y Passeron convergerían en el primer punto en la crítica de Rancière a Bourdieu. Pero en el segundo punto presentarían una crítica a Rancière en su negación de una descodificación crítica de constricciones que pesan sobre los dominados y sobre los efectos de sus relaciones diversificadas con la emancipación. En este sentido, Grignon y Passeron están más próximos a Bourdieu.

En la misma obra, Jean Claude Passeron desembarca en "la hipótesis de la ambivalencia [...] llamando a realizar una doble lectura" (1989, p. 73). Lo que es de alguna manera un llamado a que podamos tomar la problemática por las dos puntas: por las capacidades del oprimido y por su incapacidad para realizar nuevas aproximaciones críticas y comprensivo-pragmáticas. En esta perspectiva, una sociología crítica de la dominación no sería necesariamente un obstáculo a una lógica emancipadora, sino un espacio dinámico de tensiones que pueden abrirse entre ambas. Tensiones, dado que las matrices de miserabilismo y de populismo seguirían presentes y no habría punto de armonía definitiva posible entre crítica y emancipación, no habría el "fin de la historia" hegeliano.

\section{Conclusión}

La exploración de las desventuras académicas actuales de la teoría crítica en el seno de las ciencias sociales francesas como configuración entre la sociología crítica de Pierre Bourdieu y la filosofía de la emancipación de Jacques Rancière, así como los aportes de la sociología libertaria de Alfredo Errandonea, no nos ha conducido a una nueva teoría crítica con apoyos emancipatorios unificados. Sin embargo, nos ha ayudado a delimitar un espacio de problemas y a generar algunas pistas para articular cuestiones que muchas veces se presentan desarticuladas en el pensamiento y la política contemporánea.

No obstante, se trata de una articulación en el reconocimiento de tensiones. Dos pistas han sido esquematizadas: 1) una pista epistemológica de dis- 
tanciamiento comprometido que desplaza la ruptura tradicional entre juicio de hecho y juicio de valor; y 2) una pista teórica que nos incita a partir a la vez de las incapacidades y de las capacidades de los dominados. En el primer plano, el equilibrio entre distanciamiento científico y compromiso ético y político aparece inestable. En el segundo plano, los riesgos cruzados son que las incapacidades del ser se traguen las capacidades o ser insensibles a las incapacidades en la celebración de las capacidades. Los dos casos se inscriben epistemológicamente más en "la equilibración de los contrarios" enunciada por el socialista libertario Pierre-Joseph Proudhon que en "la superación de las contradicciones" de inspiración hegeliana.

\section{Referencias bibliográficas}

Boltanski, L. (2009). De la critique. Précis de sociologie de l'émancipation. París: Gallimard.

Boltanski, L. y A. Esquerre (2014). Vers l'extrême. Extension des domaines de la droite. Bellevaux: Dehors.

Boltanski, L. y N. Fraser (2016). Dominación y emancipación. Una crítica radical del capital sin nostalgia estatista. Buenos Aires: Capital Intelectual.

Bourdieu, P. (1979). La distinction. Critique sociale du jugement. París: Minuit.

Bourdieu, P. (ed.) (1993). La misère du monde. París: Seuil.

Bourdieu, P. (1997). Méditations pascaliennes. París: Seuil.

Chanial, P. (2001). Justice, don et association. La délicate essence de la démocratie. París: La Découverte.

Corcuff, P. (1991). Constructions du mouvement ouvrier. Activités cognitives, pratiques unificatrices et conflits dans un syndicat de cheminots. París: Escuela de Estudios Superiores en Ciencias Sociales (EHESS).

Corcuff, P. (2009). Pierre Bourdieu (1930-2002) leído de otra manera. Crítica social postmarxista y el problema de la singularidad individual. Cultura y Representaciones Sociales, 4(7), pp. 9-24. Disponible en: <http://www.culturayrs.org.mx/index.php/ CRS/article/view/554/561>. [acceso 5/2/2018].

Corcuff, P. (2012). Où est passée la critique sociale? Penser le global au croisement des savoirs. París: La Découverte.

Corcuff, P. (2014). Les années 30 reviennent et la gauche est dans le brouillard. París: Textuel.

Corcuff, P. (2016a). Antinomias y analogías como instrumentos transversales en sociología: a partir de Proudhon y de Passeron. Cultura y Representaciones Sociales, 10(20), pp. 42-58. <http://www.culturayrs.org.mx/index.php/CRS/article/view/342/342>. [acceso 5/2/2018].

Corcuff, P. (2016b). El político y el científico. Andamios. Revista de Investigación Social, 13(31), pp. 155-174. Disponible en: <http://www.uacm.edu.mx/Portals/18/ num31/009_Traduccion.pdf>. [acceso 5/2/2018]. 
De Lagasnerie, G. (2007). L'empire de l'université. Sur Bourdieu, les intellectuels et le journalisme. París: Éditions Amsterdam.

De Lagasnerie, G. (2016). Juger. L'État pénal face à la sociologie. París: Fayard.

Elias, N. (1993). Engagement et distanciation. Contributions à la sociologie de la connaissance. París: Fayard.

Errandonea, A. (1972). Explotación y dominación. El problema de la categoría definitoria de las clases sociales. Montevideo-Buenos Aires: Editorial Acción Directa.

Errandonea, A. (2003). Anarquismo para el siglo XXI. Revista de Ciencias Sociales, XVI (21), pp. 45-56.

Grignon, C. y J.C. Passeron (1989). Le savant et le populaire. Misérabilisme et populisme en sociologie et en littérature. París: Gallimard/Seuil.

Heinich, N. (1998). Ce que l'art fait à la sociologie. París: Minuit.

Heinich, N. (2017). Des valeurs. Une approche sociologique. París: Gallimard.

Horkheimer, M. (1996). Théorie traditionnelle et théorie critique. París: Gallimard [1937].

Kalinowski, I. (2005). Leçons wébériennes sur la science \& la propagande. En: M. Weber. La science, profession \& vocation. Marseille: Agone, pp. 61-273.

Kant, I. (1991). Réponse à la question : Qu'est-ce que les Lumières ? [1784]. En: J.-F. Poirier y F. Proust (eds.). Vers la paix perpétuelle - Que signifie s'orienter dans la pensée? Qu'est-ce que les Lumières? Et autres textes. París: Flammarion, pp. 41-45.

Lahire, B. (2016). Pour la sociologie. Et pour finir avec une prétendue "culture de l'excuse”. París: La Découverte.

Mills, C. W. (2006). L’imagination sociologique. París: La Découverte/Poche. [1959]

Prieto, R. G. (2003). La libertad como deseo, como problema y como proyecto. Revista de Ciencias Sociales, XVI(21), pp. 10-15.

Proudhon, P.-J. (1988). De la Justice dans la Révolution et dans l'Église. París: Fayard. [1858]

Proudhon, P.-J. (1997). Théorie de la propriété. París: L’Harmattan. [1866]

Rambaud, E. (2009). L'organisation sociale de la critique à Médecins sans frontières. Revue française de science politique, 59(4), pp. 723-756.

Rambaud, E. (2017). La "petite" critique, la "grande" et "la Révolution. Pour une acception non normative de la critique. Revue française de science politique, 67(3), pp. 469-495.

Rancière, J. (1983). Le philosophe et ses pauvres. París: Fayard.

Rancière, J. (1995). La Mésentente. Politique et Philosophie. París: Galilée.

Rancière, J. (2004). Le maître ignorant. Cinq leçons sur l'émancipation intellectuelle. París: 10/18. [1987]

Rancière, J. (2008). Le spectateur émancipé. París: La Fabrique.

Rancière, J. (2009). Critique de la critique du "spectacle". En: J. Rancière. Et tant pis pour les gens fatigués. Entretiens. París: Amsterdam, pp. 619-636.

Spinoza, B. (1965). Éthique. París: GF-Flammarion. [1677]

Weber, M. (1965a). L'objectivité de la connaissance dans les sciences et la politique sociales. En: M. Weber. Essais sur la théorie de la science. París: Plon, pp. 117-213. [1904] 
Weber, M. (1965b). Essai sur le sens de la " neutralité axiologique" dans les sciences sociologiques et économiques. En: M. Weber. Essais sur la théorie de la science. París: Plon, pp. 399-477. [1917]

Weber, M. (2003). La profession et la vocation de politique. En: M. Weber. Le savant et le politique. París: La Découverte. [1919]

Wschebor M. (2003). El libertario. Revista de Ciencias Sociales, XVI(21), pp. 7-9. 